\title{
Consommation De Boissons Desalterantes Et Risques Sanitaires Dans Les Colleges De La Ville D'abomey (Republique Du Benin)
}

\author{
V. B. Fanès Azalou Tingbe, Doctorat \\ Thierry Hervé Azonhe, Maître de conférences \\ Département de Géographie et Aménagement du Territoire \\ (DGAT/FASHS/UAC, Bénin) \\ Alda Yemadje, Doctorat \\ Ecole Normale Supérieure de 1'Enseignement Technique \\ (ENSET/UNSTIM, Bénin) \\ Armand Arnaud Vido, \\ Département de Géographie et Aménagement du Territoire \\ (DGAT/FASHS/UAC, Bénin)
}

Doi: 10.19044/esj.2018.v14n33p251 URL:http://dx.doi.org/10.19044/esj.2018.v14n33p251

\begin{abstract}
This research aims to analyze the health quality of thirst-quenching drinks such as fruit juices produced, sold and consumed in schools. The research was conducted on a sample of 222 learners selected in a classroom setting plus 6 randomly selected female sales representatives in schools. The microbiological analyzes were carried out on samples of different juice drinks made in the colleges. Digitization of collected data, descriptive statistics, cross tables, chi-2 tests, etc. are the statistical tools used. The results show a significant consumption of thirst-quenching drinks: hibiscus flower juice $(39.2 \%)$, water $(24.1 \%)$, fruit juice $(21.6 \%)$, local beer-based cereals (tchakpalo), $12.8 \%$ in CEG 1 and 2 of the city of Abomey. The main reasons for their consumption are related to the need for quenching, the presence of sugar and aroma in these drinks and their almost daily availability. As for the microbiological analyzes, they show a contamination of the juice units taken with a significant difference between total flora, molds, yeasts, staphylococci, etc. The contamination factors are related to the quality of the products as well as to the process of making beverages and the hygiene of the houses. Measures to improve hygiene education and improve production techniques are needed to limit the health risks of these thirst-quenching drinks.
\end{abstract}

Keywords: Bohicon, Colleges, thirst quenching drinks, hygiene, health risks 


\section{Résumé}

La présente recherche vise à analyser la qualité sanitaire des boissons désaltérantes telles que les jus de fruits produits, vendus et consommés en milieu scolaire. La recherche a été conduite sur un échantillon de 222 apprenants sélectionnés en situation de classe auxquelles s'ajoutent 6 vendeuses choisies de façon aléatoire dans les établissements scolaires. Les analyses microbiologiques ont été effectuées sur des prélèvements de différents jus de boissons effectués dans les collèges. La numérisation des données collectées, les statistiques descriptives, les tables croisées, les tests de chi-2, etc. sont les outils statistiques utilisés. Les résultats obtenus révèlent une consommation importante des boissons désaltérantes : jus de fleur d'hibiscus $(39,2 \%)$, l'eau $(24,1 \%)$, les jus de fruits $(21,6 \%)$, la bière locale à base de céréales (tchakpalo), 12,8 \% dans les CEG 1 et 2 de la ville d'Abomey. Les principales raisons de leur consommation sont relatives à la nécessité de désaltération, la présence de sucre et d'arôme dans ces boissons et leur disponibilité quasi quotidienne. Quant aux analyses microbiologiques, elles montrent une contamination des unités de jus prélevés avec une différence significative entre flore totale, moisissures, levures, staphylocoques, etc. Les facteurs de contamination sont liés aussi bien à la qualité des produits qu'au processus de fabrication des boissons et à l'hygiène des habitations. Des mesures visant l'éducation à l'hygiène et l'amélioration des techniques de production s'imposent afin de limiter les risques sanitaires de ces boissons désaltérantes.

Mots clés: Bohicon, Collèges, boissons désaltérantes, hygiène, risques sanitaires

\section{Introduction}

Les villes africaines connaissent depuis plusieurs années une poussée démographique et un essor économique important. Il s'ensuit le développement de plusieurs activités de restauration, étant donné que les habitants se retrouvent dans l'obligation de s'assurer une restauration à prix relativement bas hors du domicile familiale (Canet et al., 1996 ; Chauliac et al., 1998) avec tous les risques de diarrhées microbiennes et de gastroentérites (Owhe et al., 1996 ; Barro et al., 2002 ; Malvy et $a l ., 1995$ ) que comporte ce type d'alimentation. La situation est identique en milieu scolaire au Bénin.

Face à la difficulté, voire l'impossibilité de rentrer se restaurer dans le cadre familial aux heures de pause, les élèves se rabattent alors sur les cantines ou autres lieux de restauration au sein de leur établissement ou dans les alentours. Ce sont ainsi des milliers d'enfants béninois qui consomment des aliments préparés avec des risques importants pour la santé. 
Pour satisfaire les besoins en eau de l'organisme, plusieurs alternatives sont offertes aux élèves. Parmi les boissons utilisées pour s'hydrater, certaines occupent une place de choix. Il s'agit principalement des jus de différents fruits, feuilles ou fleur comme l'Hibiscus sabdariffa, encore appelé oseille de Guinée ou bissap, l'ananas ou le citron. L'eau ensachée communément appelée "pure water" constitue également une source d'hydratation pour les élèves. Ces aliments prêts à être consommés sont souvent classés dans le groupe des aliments de rue définit comme étant des aliments prêts à être consommés, préparés et vendus par de vendeuses ou des colporteurs surtout dans les rues et les lieux publics (Baba Moussa et al., 2006 ; Barro et al., 2002).

Mais les conditions peu hygiéniques de préparation de ces aliments font courir des risques aboutissant parfois à leur contamination microbienne (Baba Moussa et al., 2006). En effet, les matériels de préparation, la propreté du site, l'hygiène individuelle et la salubrité de l'habitation sont autant de facteurs de risques de contamination des produits qui peuvent influer négativement sur la qualité microbiologique des boissons vendues en milieu scolaire. La conséquence est l'intoxication alimentaire fréquente, avec des effets parfois difficilement gérables (Banque mondiale, 1993).

En effet, la plupart du temps, l'intoxication alimentaire résulte de l'ingestion d'aliments contaminés par un microorganisme nocif ou un agent pathogène. Dans certains cas, la pathologie n'est pas due à la prolifération d'un microorganisme dans l'aliment mais à l'ingestion d'une toxine sécrétée par la bactérie, et préformée dans l'aliment avant son ingestion ; on parle alors d'intoxication (Marty, 1998). Dans les pays en voie de développement, les infections alimentaires demeurent un gros problème de santé publique puisqu'elles peuvent atteindre une population très importante (Malvy et Djossou, 2009). Il s'agit dans ce cas d'une toxi-infection alimentaire collective (TIAC). Les microorganismes pouvant causés des toxi-infections alimentaires sont les virus, les parasites et les bactéries. On peut retenir entre autres, Clostridium botulinum; Clostridium perfringens; Campylobacter ; Escherichia coli (notamment le 0157:H7) ; Salmonella ; et les staphylocoques (Staphylococcus aureus notamment). Dans l'alimentation, plusieurs produits peuvent être responsables des infections alimentaires et différents facteurs de risques peuvent y être rattachés ; notamment ceux d'hygiènes.

Si l'on se réfère aux cas d'intoxications de la population scolaire et aux causes probables, il est nécessaire de s'interroger sur les conditions de préparation et la qualité des boissons consommées par les élèves. Il se pose alors l'importante question des conditions et de la qualité des produits alimentaires consommés hors domicile notamment en ce qui concerne les élèves dans les écoles et collèges du Bénin. La présente recherche vise à 
étudier la qualité microbiologique des boissons désaltérantes consommées par les élèves des collèges d'enseignement général 1 et 2 de la ville d'Abomey.

\section{Milieu de recherche}

Capitale historique de la République du Bénin et chef-lieu du département du Zou, la commune d'Abomey est localisée dans la partie sud du Bénin à environ $145 \mathrm{~km}$ de Cotonou. Située entre $7^{\circ} 10^{\prime}$ et $7^{\circ} 16^{\prime}$ de latitude nord, et $1^{\circ} 52^{\prime}$ et $2^{\circ} 03^{\prime}$ de longitude est, elle est d'une densité de 552 habitants au $\mathrm{km}^{2}$ et couvre une superficie de $142 \mathrm{~km}^{2}$ avec une population de 92266 habitants soit 52,8 \% de femmes pour 47,2 \% d'hommes selon le recensement de 2012 (INSAE, 2013). Elle a pour limites, la commune de Djidja au nord, celle d'Agbangnizoun au sud, à l'est celle de Bohicon et le département du Couffo précisément la commune d'Aplahoué à l'ouest. La commune d'Abomey regroupe, selon le découpage administratif, onze (11) quartiers et dix-huit (18) villages répartis au sein de sept (07) arrondissements dont trois (03) centraux à caractère urbain que sont Djègbé, Hounli et Vidolé et quatre (04) périphériques à caractère rural que sont Agbokpa, Détohou, Sèhoun et Zounzonmè. La figure 1 représente la carte de localisation de la zone d'étude.

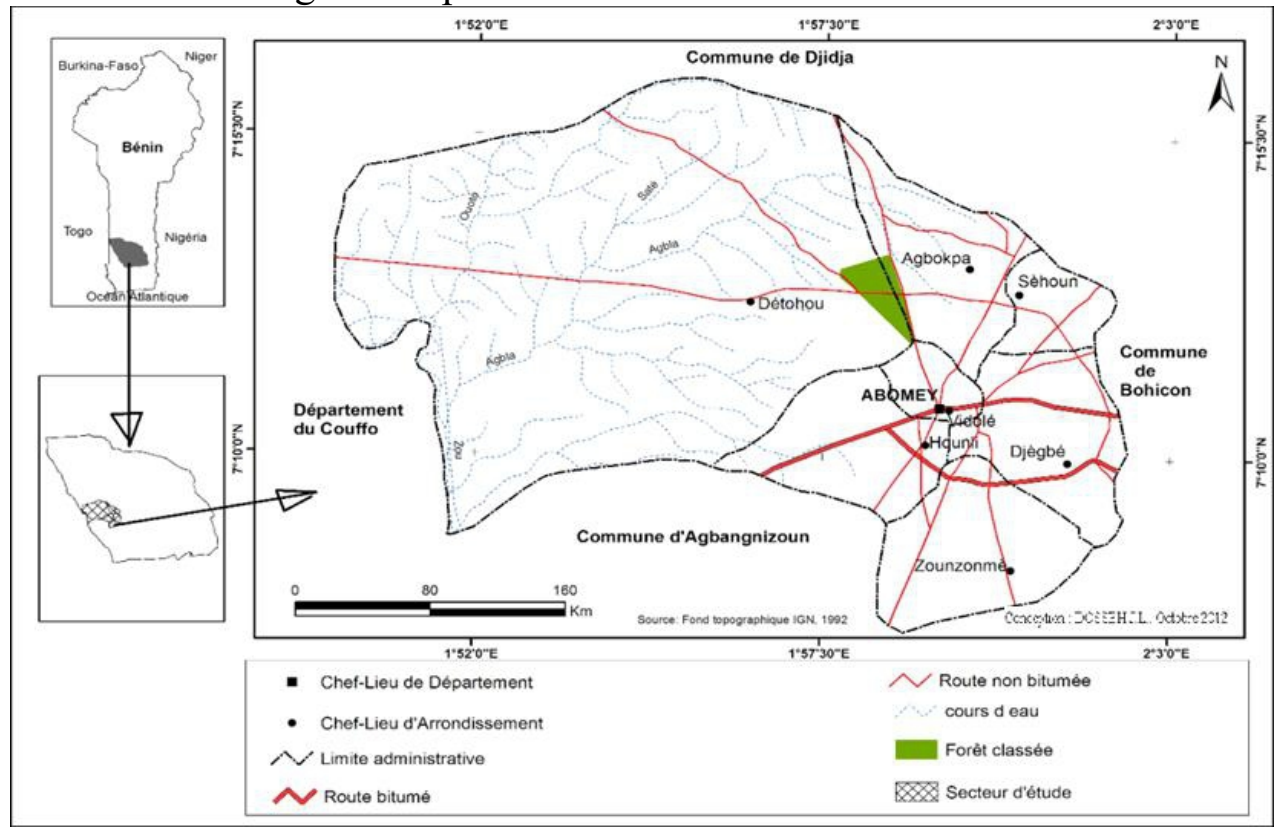

Figure 1 : Localisation géographique du milieu de recherche Source : Fond de carte topographique, IGN, 1992.

Le relief de la commune est caractérisé par un plateau au centre, avec un dénivelé d'environ $157 \mathrm{~m}$ entre le point le plus haut situé au centre de la commune et le point le plus bas dans la partie Ouest. Ainsi, les pentes y sont généralement de moins de $5 \%$ (Mairie d'Abomey, 2012). De par sa position 
géographique, elle jouit d'un climat de transition entre le climat subéquatorial de la côte et le climat tropical humide de type soudano guinéen du Nord Bénin avec une pluviométrie moyenne et annuelle de $1000 \mathrm{~mm}$. Ce type de climat, chaud et humide, influe négativement sur l'état de santé de la population, car il engendre ou favorise le développement d'agents pathogènes (Anopheles gambiae et Anopheles funestus, bactéries, etc.) responsables de la prolifération d'affections telles que le paludisme, les IRA, les dermatoses, etc. En effet, durant les saisons sèches, Anopheles funestus remplacerait Anopheles gambiae dans le maintien de l'endémisme du paludisme (Azonhè, 2009).

La ville d'Abomey est une création de la dynastie des Alladahonou, creuset de la civilisation Fon. Elle s'est développée à partir des palais royaux avec des populations essentiellement Fon auxquelles se sont ajoutées d'autres ethnies (Mahi, Haoussa, Goun, Yoruba, Dendi, Peulh, Somba, Mina, Adja, etc.) pour des raisons guerrières serviles ou commerciales. La ville est essentiellement peuplée de natifs Fon représentant $98 \%$ de la population qui, ancienne et spécifique, croit à un taux annuel moyen de 2,2 \% (INSAE, 2013).

Selon le RGPH4 (INSAE, 2013), les principales activités de la population d'Abomey sont, par ordre d'importance décroissante, le commerce (46\% des actifs), l'artisanat (17\%), le fonctionnariat (15\%) et l'agriculture $(11 \%)$. Le nombre d'actifs dans le secteur agricole a diminué de plus de moitié entre 1979 et 2002 (en passant de $24 \%$ à $11 \%$ ) au profit du commerce pendant que le nombre des artisans est resté stable dans la même période (Monographie-Abomey, 2006). En 2012, les travaux du recensement révèlent une prédominance des commerçants, des fonctionnaires et des artisans (Mairie d'Abomey, 2012). Il est à noter que la présence du grand marché central Houndjro et ses annexes comme le marché Adjahito assurent à la ville une fonction de grand centre de commerce traditionnel. Aussi existe-t-il dans le centre-ville et à la périphérie de la ville, des boutiques, des buvettes et des mini-supermarchés qui gravitent autour du marché Houndjro.

\section{Données et méthodes}

La recherche est de nature transversale à visée descriptive et analytique, ayant nécessité l'utilisation de plusieurs données comme : les données sociodémographiques, les statistiques scolaires, les données épidémiologiques, les données physicochimiques et bactériologiques obtenues au laboratoire pour rechercher la présence de germes pathogènes dans les boissons vendues dans les CEG ciblés.

La population d'étude est constituée des adolescents scolarisés et l'ensemble des vendeuses de boissons dans les collèges d'enseignement général 1 et 2 de la ville d'Abomey. Les élèves ont été sélectionnés selon une technique de sondage aléatoire à deux degrés. La base de sondage étant la population de ces deux collèges. Un calcul proportionnel à la taille de chaque 
établissement du nombre d'élèves à enquêter dans chacun des collèges sélectionnés a été effectué. La taille minimale de l'échantillon calculée selon la formule de Schwartz (1995) est de 222 individus, auxquels s'ajoutent 6 vendeuses de boissons.

La collecte des données s'est effectuée avec les entretiens individuels sur la base d'un questionnaire pour les élèves et les enseignants, et des entretiens de groupe avec les vendeuses de boissons dans les collèges 1 et 2 d'Abomey. L'observation directe a permis d'appréhender sur les sites de préparation de ces boissons, les risques liés aux différentes étapes du processus de fabrication. Le traitement des données a consisté au dépouillement minutieux fondé rigoureusement sur la spatialisation des informations recueillies. Les fiches sont vérifiées, codées puis numérisées grâce au logiciel EPIDATA 3.1. Les données saisies ont été importées dans le logiciel statistique SPSS 17.1 pour leur traitement.

S'agissant des analyses physicochimiques et bactériologiques, les prélèvements ont été faits à $10 \mathrm{~h}$ et à $16 \mathrm{~h}$. Le choix de ces tranches horaires découle du fait qu'il s'agit des heures de recréation des enfants. De plus, généralement préparé la veille, le jus, dans les conditions de préparation et de conservation a largement le temps de connaitre un début de contamination au lendemain de sa préparation. Ce qui veut dire que forcément, au plan bactériologique, il y a une différence entre le jus fraichement préparé et le jus servi à l'école pour désaltérer les enfants. Les conditions de conservation du jus à l'école et les différentes manipulations justifient aussi le choix de la deuxième heure de recréation. Ainsi, les chances d'obtenir des résultats fiables quant à la qualité microbiologique des jus consommés sont grandes. Le nombre de prélèvement dans les établissements couverts s'élève à 6 . Les produits prélevés sont le Adoyo, le jus de bissap et le tchakpalo. Ces prélèvements ont été faits auprès des vendeuses des boissons désaltérantes en situation de commercialisation (vente) à des heures différentes de la journée. Pour réaliser les prélèvements, les matériels suivants ont été utilisés :

$>$ Un pH- mètre WTW 315I/SET ;

$>$ des flacons de prélèvements en verre borosilicate ;

$>$ une glacière ;

$>$ des condensateurs de froids.

La recherche et le dénombrement des germes s'est fait au laboratoire.

\section{Résultats et analyses}

\section{Consommation de boissons en milieu scolaire}

La consommation de boissons désaltérantes est une réalité en milieu scolaire comme le révèle la figure 2 . 


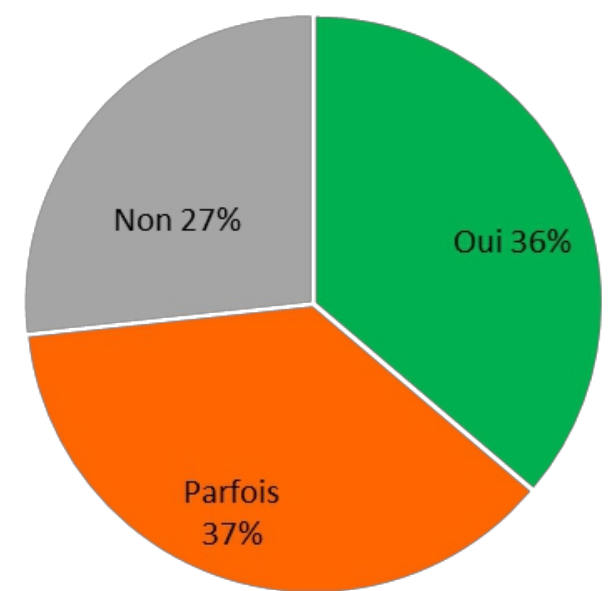

Figure 2 : Consommation régulière de boissons pendant la récréation Source : données de terrain, janvier 2017.

Il ressort de la figure 2 que $37 \%$ des enquêtés déclarent ne pas consommer régulièrement une boisson, l'eau y compris pendant la récréation. Les motifs de cette absence de régularité ne sont pas identifiés. Cependant 36 $\%$ déclarent consommer régulièrement les jus vendus à l'école, contre $27 \%$ qui n'en consomment pas du tout. Parmi ceux qui ont déclaré qu'ils consommaient des boissons désaltérantes au cours de la récréation, leurs préférences ont été explorées et se résument dans la figure 3.

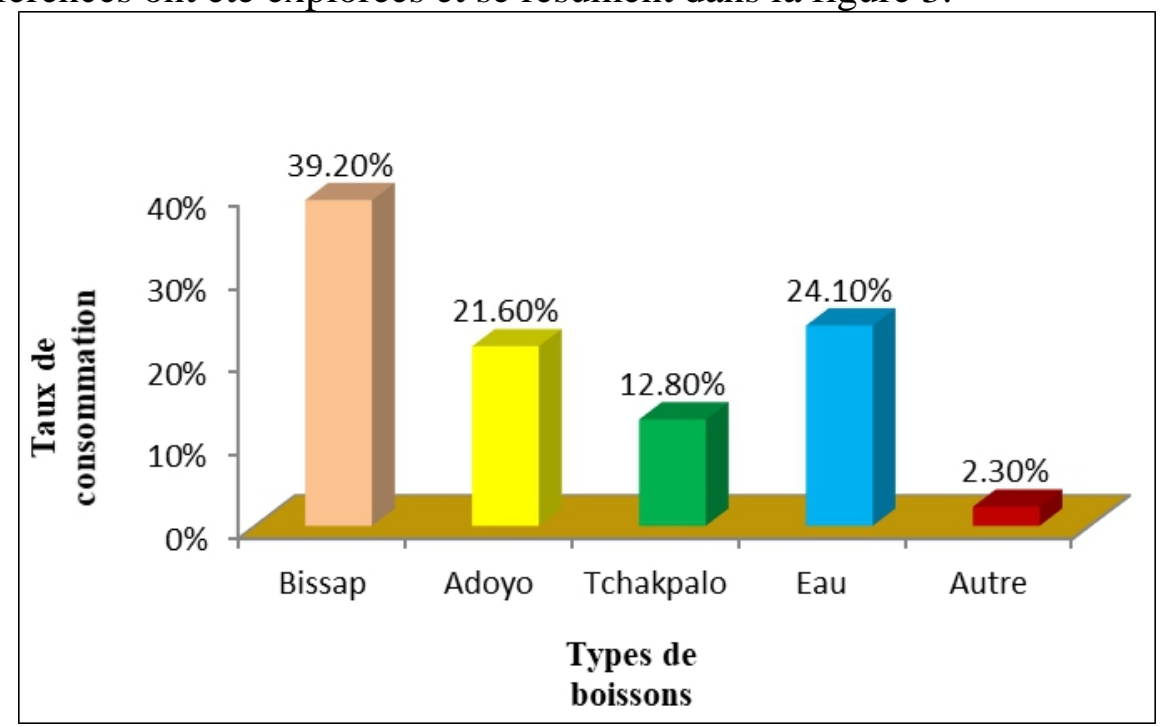

Figure 3 : Boissons consommées régulièrement par les élèves Source : données de terrain, janvier 2017.

Il découle de la figure 3 que le jus de bissap est la boisson de préférence de $39,2 \%$ des élèves. Viennent ensuite l'eau $(24,1 \%)$ et le jus adoyo $(21,6$ 
$\%)$. Le tchakpalo reste la boisson la moins consommée (12,8\%) suivi d'autres jus (Fan Milk, Fan Ice, etc.) qui fait moins de $3 \%$. Le jus adoyo est une boisson issue de l'eau provenant de la fermentation du maïs non germé, sucrée et glacée. Le tchakpalo est une bière de mil traditionnelle et le jus de bissap est une boisson préparée avec des fleurs d'hibiscus rouges cuites au sirop. Quant à la fréquence de consommation des boissons dans la journée, les résultats sont présentés dans la figure 4.

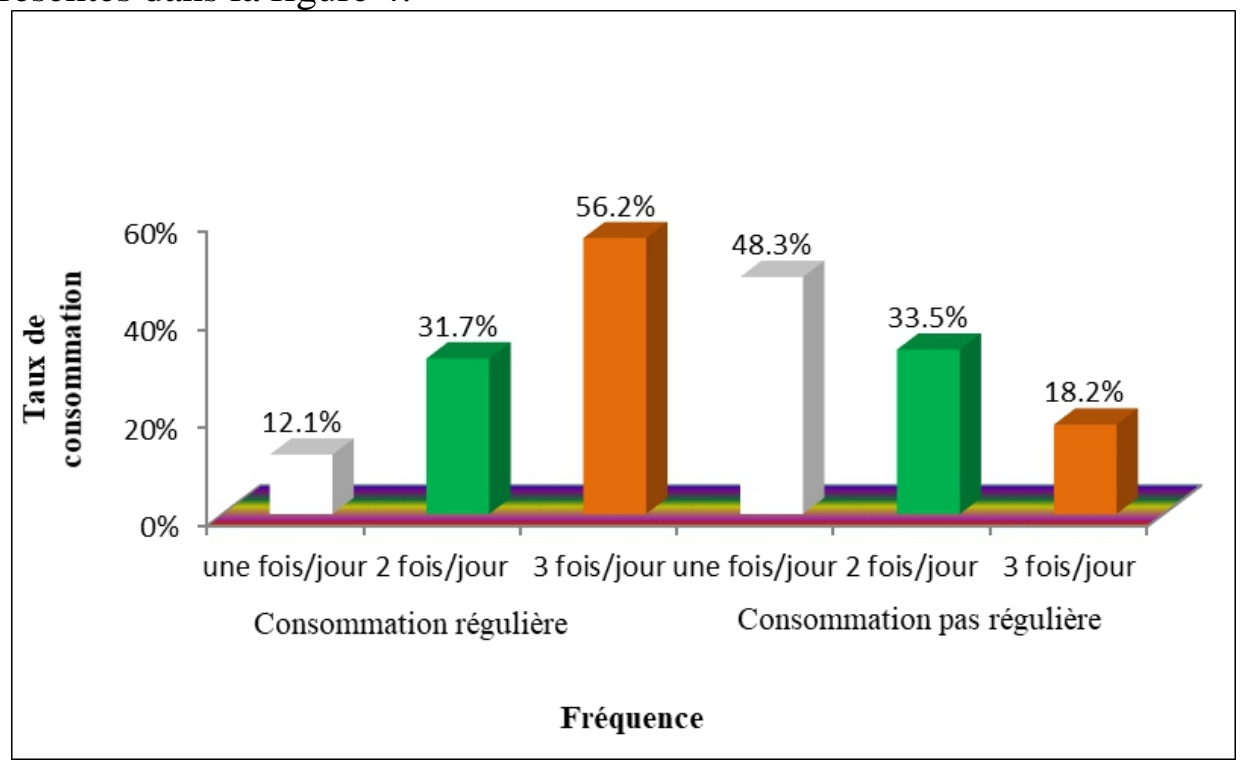

Figure 4 : Fréquence de consommation des boissons désaltérantes en milieu scolaire Source : données de terrain, janvier 2017

La fréquence de consommation de ces différentes boissons montre que chez les consommateurs réguliers, la triple consommation (consommation trois fois par jour) est plus élevée $(56,2 \%)$ que la consommation unique qui ne concerne que $12,1 \%$ des élèves. Chez les consommateurs irréguliers, la prise de ces boissons est unique pour presque la majorité du sous échantillon, soit 48,3\% contre 33,5\% pour ceux qui consomment deux fois par jours. Cependant, les motifs qui justifient la régularité de la consommation de ces boissons sont multiples et sont moins du fait de leur teneur en sucre que de l'envie de boire comme le révèle la figure 5 . 


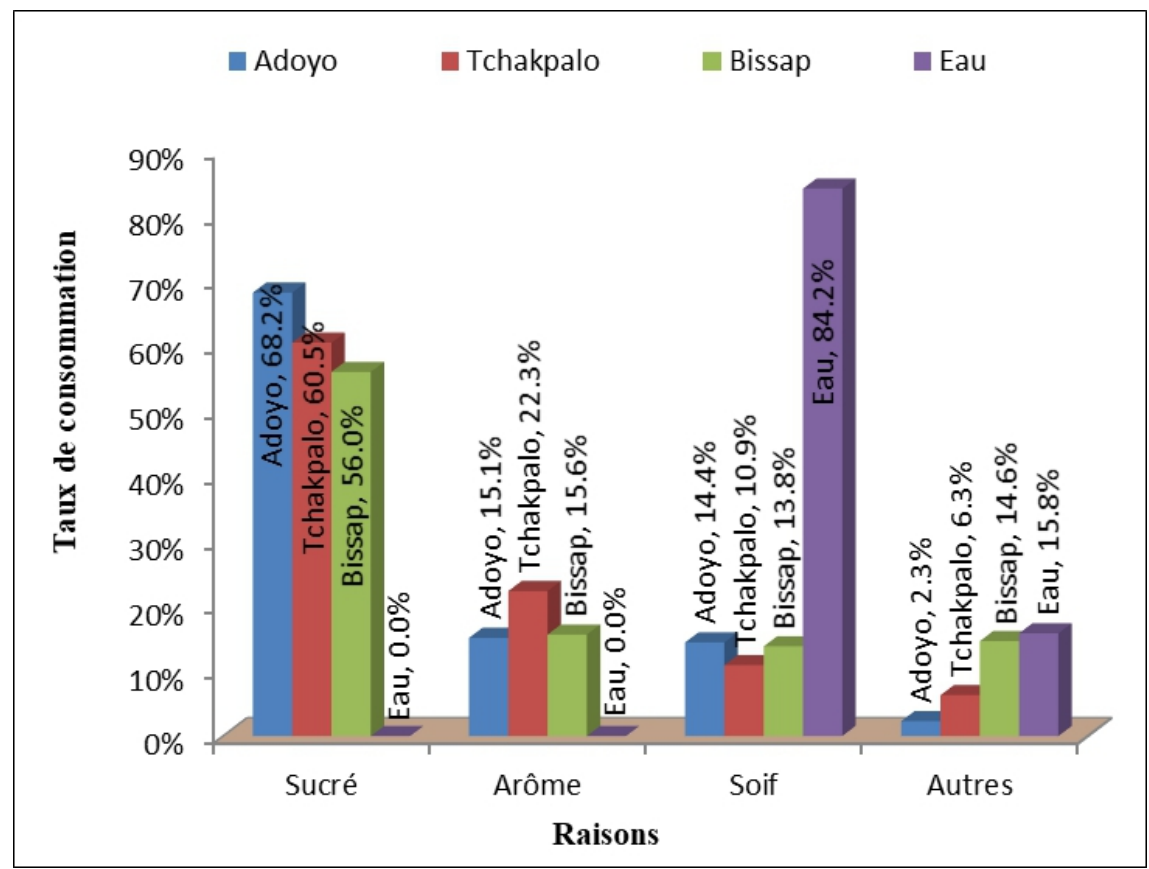

Figure 5 : Raisons de la consommation régulière des différentes boissons Source : données de terrain, janvier 2017.

De l'analyse de la figure 5, il ressort qu'en dehors de l'eau (84,2\%) surtout consommée pour étancher la soif, la consommation de sucre est le motif principal avancé par les élèves pour justifier la consommation de ces boissons. Ainsi, le Tchakpalo, le Adoyo, le Bissap et les autres comme le Fan Milk et la sucette sont surtout consommés pour leur arômes et pour le sucre.

\section{Qualité des boissons consommées en milieu scolaire}

L'analyse des échantillons de jus prélevés lors des différents passages dans les établissements scolaires retenus donne les résultats présentés dans le tableau I.

Tableau I : Résultats d'analyses bactériologiques des prélèvements de jus vendus dans les établissements scolaires à 10h (matinée)

\begin{tabular}{|c|c|c|c|c|c|c|c|c|c|}
\hline \multicolumn{10}{|c|}{ Prélèvement de $10 \mathrm{~h}$} \\
\hline Collèges & Produits & $\begin{array}{l}\text { Flore totale } \\
\text { UFC/ml }\end{array}$ & $\begin{array}{l}\text { Coliformes } \\
\text { totaux } \\
\text { UFC/ml }\end{array}$ & $\begin{array}{l}\text { Coliformes } \\
\text { fécaux } \\
\text { UFC/ml }\end{array}$ & \begin{tabular}{|l|} 
E. Coli \\
UFC/ml
\end{tabular} & $\begin{array}{l}\text { Staph. } \\
\text { Aureus } \\
\text { UFC/ml }\end{array}$ & ASR & $\begin{array}{l}\text { Levures } \\
\mathrm{UFC} / \mathrm{ml}\end{array}$ & $\begin{array}{l}\text { Moisissures } \\
\text { UFC/ml }\end{array}$ \\
\hline \multirow{3}{*}{ CEG 1} & Adoyo & - & 6 & 1 & - & 10 & 10 & 220000 & 1000 \\
\hline & Bissap & 30000000 & 1 & 1 & - & 10 & 10 & 30000000 & 1000 \\
\hline & Tchapalo & & & & - & & & & \\
\hline \multirow{3}{*}{ CEG 2} & Adoyo & - & 370 & 47 & - & 10 & 10 & 860000 & 1000 \\
\hline & Bissap & 3000000 & 320 & 7 & - & 10 & 10 & 30000000 & 1000 \\
\hline & Tchapalo & - & 48 & 1 & - & 10 & 10 & 30000000 & 1000 \\
\hline
\end{tabular}


Tableau II : Résultats d'analyse bactériologique des jus vendus dans les établissements scolaires à $16 \mathrm{~h}$ (soirée)

\begin{tabular}{|c|c|c|c|c|c|c|c|c|c|}
\hline \multicolumn{10}{|c|}{ Prélèvement de 16 h 30} \\
\hline Collèges & Produits & $\begin{array}{l}\text { Flore totale } \\
\text { UFC/ml }\end{array}$ & $\begin{array}{l}\text { Coliformes } \\
\text { totaux } \\
\text { UFC/ml }\end{array}$ & $\begin{array}{l}\text { Coliformes } \\
\text { fécaux } \\
\text { UFC/ml }\end{array}$ & $\begin{array}{l}\text { E. Coli } \\
\text { UFC/ml }\end{array}$ & $\begin{array}{l}\text { Staph. } \\
\text { Aureus } \\
\text { UFC/ml }\end{array}$ & ASR & \begin{tabular}{|l} 
Levures \\
UFC/ml
\end{tabular} & $\begin{array}{l}\text { Moisissures } \\
\text { UFC/ml }\end{array}$ \\
\hline \multirow{3}{*}{ CEG 1} & Adoyo & - & 170 & 2 & - & 10 & 10 & 3000000 & 100000 \\
\hline & Bissap & 3200 & 1 & 1 & - & 10 & 10 & 1000 & 1000 \\
\hline & Tchapalo & & & & - & & & & \\
\hline \multirow{3}{*}{ CEG 2} & Adoyo & - & 10 & 10 & - & 10 & 10 & 7200 & 100 \\
\hline & Bissap & 3000000 & 10 & 10 & - & 10 & 10 & 3000000 & 28000 \\
\hline & Tchapalo & & 10 & 10 & - & 10 & 10 & 3000000 & 100 \\
\hline
\end{tabular}

L'analyse des tableaux I et II révèle des résultats intéressants. On constate dans un premier temps l'absence d'Escherichia coli dans tous les échantillons des boissons prélevées aussi bien à $10 \mathrm{~h}$ qu'à $16 \mathrm{~h} 30 \mathrm{mn}$. Le nombre des anaérobies sulfito réducteurs (ASR) est inférieur ou égal à 10 UFC/ml. Le jus de bissap est la boisson qui contient le plus de germes avec une flore totale qui varie entre $3,2.103$ et $3.107 \mathrm{UFC} / \mathrm{ml}$. Les variations au niveau de tous les germes sont très importantes passant parfois du simple à plus du double notamment au niveau des coliformes. Avec la présence en nombre élevé des levures et moisissures également, on peut conclure à une contamination bactériologique des boissons vendues en milieu scolaire. Cette situation observée au premier passage n'est pas fondamentalement différente de celle observée au second passage durant laquelle la même contamination a été observée notamment pour le jus de bissap mais avec une variation du nombre de germes.

En outre, la comparaison inter collège ne révèle pas une différence significative. En effet, il apparaît que le jus de bissap est la boisson qui contient le plus de germes dans tous les collèges avec la valeur la plus élevée obtenue au CEG 2 (3000000 UFC/ml). On observe que des germes se retrouvent dans tous les collèges sauf que le jus de tchapalo vendu au CEG 1 ne contient aucun germe.

La probabilité de présence de germes pathogènes intestinaux croît parallèlement au degré de pollution et à la prévalence de maladies à transport hydrique qui contribue à la pollution de certaines sources. Pour cette raison, des bactéries indicatrices de contamination fécale sélectionnées sont celles du groupe des coliformes, c'est-à-dire des bactéries provenant du colon ou du gros intestin des animaux et de l'homme. Outre ce groupe de bactéries, on retient également Salmonella typhosa, agent causal de la fièvre typhoïde comme un indicateur important de la contamination bactériologique. Les résultats des analyses bactériologiques indiquent que le risque de toxi infection alimentaire est assez important. Le deuxième passage n'a pas donné des résultats plus 
intéressants, justifiant de ce fait, la faible application des normes d'hygiène dans le processus de fabrication des boissons et de leur distribution.

Une analyse plus profonde permet d'observer que la présence constante de la Flore mésophile aérobie totale et des Coliformes thermotolérants (CT) dans les échantillons est due sans doute à l'environnement. En effet, les conditions climatiques et environnementales des régions intertropicales sont favorables à la multiplication végétative des cellules microbiennes (Azonhè, 2009). Le séjour prolongé à la température ambiante ainsi que l'exposition à l'air libre constitue deux facteurs majeurs de contamination et de multiplication de la Flore Mésophile Aérobie Totale dans les aliments de la rue. Quant aux staphylocoques, leur présence tient souvent de l'action des facteurs tels que : le vent, la poussière et aussi la contamination d'origine humaine à travers les manipulations et les sécrétions (salive, sueur). Pour ce qui est de la flore fongique, sa présence est due à l'atmosphère humide des locaux où s'effectuent la préparation et la vente des aliments (Fayomi et al., 1992). On peut incriminer également l'inefficacité des méthodes de conservation des aliments. La présence des salmonelles et des anaérobiesulfito-réducteurs (ASR) est due à l'inefficacité de l'effet thermique bactéricide de la température de cuisson (Jouve, 1996, 2006). Sachant en effet que, la contamination des aliments à diverses origines (originelle : viande provenant d'animaux malades ou porteurs ; directe par des individus porteurs ou malades et enfin indirecte : contact des aliments avec un milieu pollué au cours de la préparation). On peut catégoriser en quelques points les origines probables de la contamination à travers le suivi du processus de fabrication des boissons chez quelques vendeuses.

\section{Risques de contamination liés au processus de fabrication des jus}

En fonction de la boisson à préparer, divers ustensiles sont utilisés. Il s'agit des bassines, du mortier avec son pilon ou une palette de cuisine; des sceaux et des bols. Les ustensiles sont généralement rincés à l'eau propre et disposés au sol contre un mur pour les égoutter. Dans certains cas, la marmite de cuisson est celle utilisée pour la cuisine dans le ménage. Les cannettes de bières sont préalablement lavées. Mais les sachets ne le sont plus.

\section{Préparation du jus de bissap}

L'ananas découpé en petites tranches avec la peau est pilé dans la marmite ou le mortier avec un pilon afin de bien en extraire le jus. Les fleurs de bissap non lavées sont ajoutées à l'ananas pilé (Planche 1). Une grande quantité d'eau y est ajoutée et le tout est porté à ébullition. Le mélange bouilli est déversé dans un récipient recouvert d'un tissu à maille fine pour la filtration du jus. Le mélange est remis sur le feu avec de l'eau pour une deuxième et une 
troisième préparation. Le jus obtenu est laisser à l'air libre dans des bassines afin d'accélérer le refroidissement.

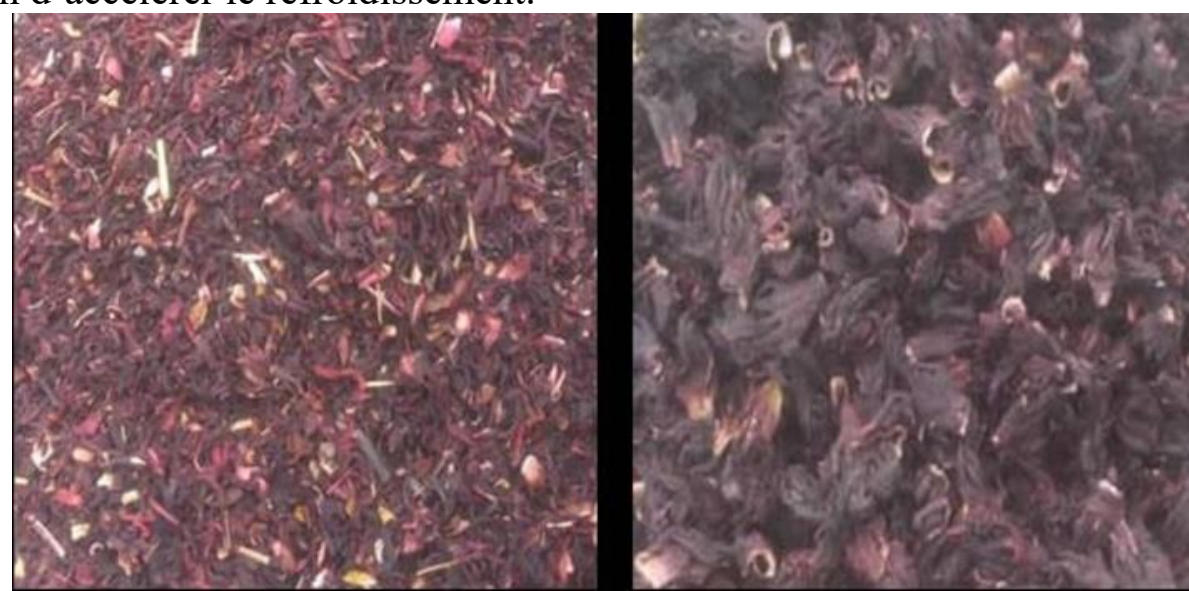

Planche 1 : Calices séchés de deux variétés (Koor) d'Hibiscus sabdariffa Prise de vue : Vido A., Janvier 2017.

Les fleurs d'Hibiscus sabdariffa encore appelées oseille de Guinée ou encore bissap, auraient des propriétés hématologiques intéressantes, ce qui favorise la consommation du jus de bissap. Les fruits utilisés ne sont donc pas lavés. La situation au niveau des fleurs de bissap est encore pire. En effet, toute humidification des fleurs dans un liquide entraîne forcément la perte d'une partie de sa concentration rouge ; ce qui diminue la valeur marchande du jus qui serait préparé. On observe donc une insuffisance de l'hygiène dans le nettoyage des matières premières et des ustensiles de cuisine.

\section{Préparation du tchakpalo}

Pour les élèves au sein des collèges 1 et 2 d'Abomey, le produit vendu est du «Tchakpalo»: boisson fermentée obtenue à partir du maïs en germination, sucrée et glacée, de couleur marron, elle est très appréciée. En effet, les vendeuses font bouillir l'eau de maïs fermenté avec des feuilles de citronnelle. Cette préparation refroidie est mélangée à du caramel (sucre calciné) pour lui donner la coloration marron proche de celle du « Tchakpalo ». Le tout est laissé à l'air libre, aux mouches, aux insectes, etc. pour refroidissement avant le conditionnement.

La durée de cuisson de chaque produit est variable. Dans tous les cas, pour économiser l'énergie, le produit est supposé cuit lorsqu'il est en ébullition puisqu'une trop longue cuisson pourrait également dénaturer le jus. Ainsi, plusieurs germes pathogènes résistent à cette chaleur et continuent d'être vivaces dans le jus commercialisé. Le jus refroidi est déposé dans la chambre à coucher jusqu'au lendemain matin où sans chauffage, il est transporté à l'école il y a là encore, plusieurs risques de contamination dus à 
l'insalubrité des concessions et à la pollution de l'air intérieur. Le reste du jus, généralement non sucré, est conservé dans un autre bidon dans le magasin à l'école, mélangé avec la nouvelle préparation pour être revendu. Pour éviter la fermentation, le jus conservé n'est pas sucré.

Le conditionnement commence généralement vers $19 \mathrm{~h}$ afin d'éviter les mouches. Le jus refroidi est sucré et à l'aide d'un bol, les canettes et les sachets sont remplis. Les canettes et les sachets sont mis au congélateur jusqu'au lendemain si l'appareil est disponible. Si l'appareil n'est pas disponible, les sachets sont conservés dans une bassine jusqu'au lendemain. Ils sont ensuite transvasés dans une glacière et on y ajoute de la glace. Dans le cas où la vendeuse ne dispose ni de glacière, ni de congélateur, elle verse le jus préparé et refroidi dans des bidons. Il sera sucré et glacé à l'école. La plupart des vendeuses utilisent cette forme de conservation (planche 2).
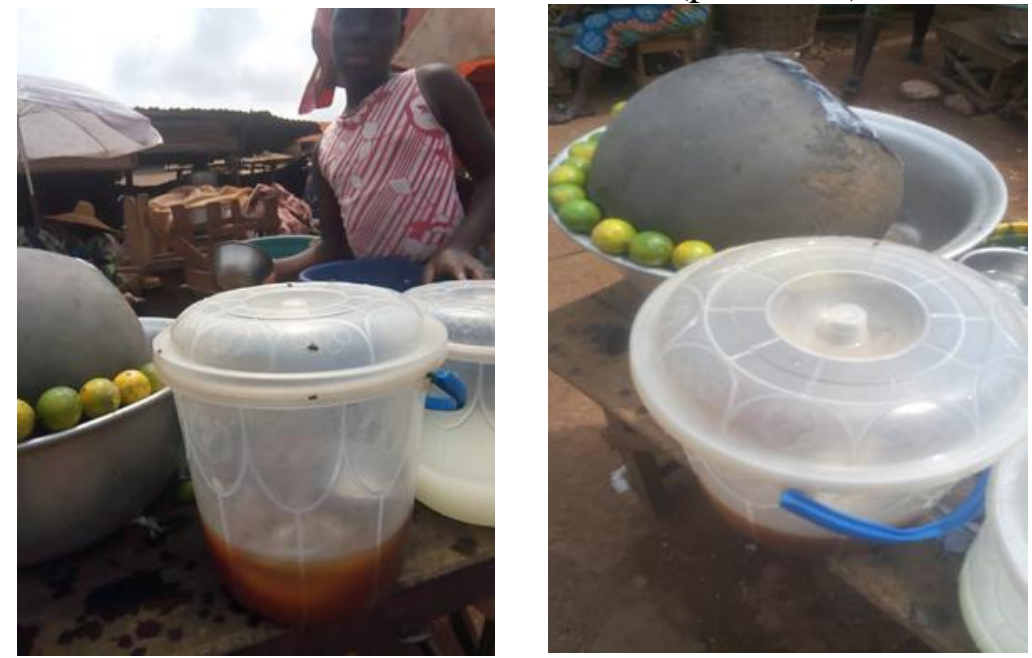

Planche 2: Conditions de conservation et de vente des jus de tchakpalo et de adoyo Prise de vue, Vido A., Janvier 2017.

\section{Risques liés aux conditions d'habitation, aux matériels et aux produits}

Il apparaît selon les observations que les jus vendus sont loin d'offrir toutes les garanties de salubrité. Ils sont impropres à la consommation de par les conditions d'hygiène qui entourent le processus de fabrication, (provenance de l'eau, qualité des intrants, conditions de préparation, etc.) ; de par le manque d'hygiène lors de son conditionnement (contenants souillés, mains sales, poussières) ou en raison de la nature de l'habitation et des sites de fabrication. Des visites effectuées chez les femmes vendeuses de boissons, révèlent qu'aucune d'elles ne disposaient d'un site particulier affecté à la préparation des boissons. Dans la plupart des cas, ces sites sont confondus avec la cour de la maison et la cuisine du ménage en ce qui concerne le chauffage. La cour ou la véranda sont les espaces généralement affectés aux principales étapes du processus. 
De ce fait, si l'on suit la filière partant des matières premières jusqu'aux produits finis, réside le problème du respect strict des règles d'hygiène, la conservation des aliments. L'exposition des aliments à la température ambiante favorise la prolifération des germes à l'origine de l'altération et des risques d'intoxication alimentaire.

Au niveau de l'acquisition des différents intrants, on observe en ce qui concerne les fruits que les soucis d'économie et d'accroissement des bénéfices amènent des vendeuses à acheter des fruits de qualité douteuse. Beaucoup de fruits acquis par les vendeuses sont souvent en début de détérioration avec ainsi un nombre important de microorganismes (responsables de la dégradation des fruits matures). Il a été observé que dans la préparation du jus d'ananas par exemple, le fruit est juste rincé et non lavé à l'eau et au savon.

\section{Discussion}

Les jus de boissons vendus dans les collèges d'Abomey subissent lors $\mathrm{du}$ processus de fabrication et de vente des opérations peu hygiéniques aboutissant pour la plupart à des contaminations microbiennes. C'est ce que soulignaient déjà les travaux de Baba-Moussa et al. (2006) qui ont fait observer que les produits qui ne sont pas soumis au chauffage lors de la préparation ont les charges microbiennes les plus élevées. Les conditions de préparation et la qualité des boissons consommées par les élèves présentent des risques d'intoxications et des affections gastro-entérites dans la population scolaire. Ces résultats confirment ceux obtenus par Chauliac et al. (1998) dans la ville de Cotonou et Fayomi et al. (1992) en milieu rural béninois.

Au niveau de la vente des jus de boissons, le jus préparé n'est plus chauffé avant d'être transporté à l'école. Ces résultats corroborent ceux obtenus par Baba-Moussa et al. (2006). En effet, le jus refroidi et celui de la veille conservé dans un bidon au magasin de l'école ne sont plus réchauffés avant la vente, ce qui accroît les risques de contamination.

Les résultats de la recherche ont montré que le jus de bissap est la boisson qui contient le plus de germes dans tous les collèges. La probabilité de présence de germes pathogènes intestinaux croit parallèlement au degré de pollution et à la prévalence de maladies à transport hydrique qui contribue à la pollution de certaines sources. Les analyses bactériologiques indiquent que le risque de toxi-infection alimentaire est assez important (OMS, 2010). Monhounvedo (2016) qui a mené sa recherche sur la qualité du jus de bissap commercialisé dans la commune d'Athiémé était parvenu aux mêmes conclusions.

De même, au cours de la vente, les bols sont laissés en permanence dans un seau rempli d'eau. Après usage par un élève, le bol est retourné dans le même seau d'eau pour y être rincé. Les risques pour la santé se situent au niveau du seau d'eau dans lequel la vendeuse plonge la main tout le temps et 
y rince en permanence les bols. Si l'on admet qu'à force de circulation, les pièces de monnaies peuvent collecter des microorganismes pathogènes, alors le risque de contamination du jus vendu s'élève au fur et à mesure qu'il est vendu. En effet, la main qui sert à servir le jus aux élèves est celle qui recueille l'argent et plonge dans le seau pour rincer le bol sans compter les risques liés à l'utilisation du même bol par plusieurs élèves. Cette pratique peut faciliter la contamination puisque l'argent passe de mains en mains. Ces résultats sont conformes à ceux obtenus par Baba-Moussa et al. (2006) et Barro (2002) qui observent que beaucoup de vendeuses servent la nourriture à la main, ce qui peut entraîner des contaminations bactériennes inhérentes à la salubrité des mains. Il en est de même de Tjoa et al. (1977), Owhe et al. (1993) et Umoh et al. (1999) qui ont souligné que les mains véhiculent la plupart du temps les germes de contaminations fécales qui sont souvent responsable des gastroentérites et des maladies diarrhéiques.

\section{Conclusion}

Les jus de fleur d'oseille de Guinée ou bissap, l'ananas ou le citron, ou encore le tchakpalo, de par leurs propriétés nutritionnelles et médicinales et leur disponibilité sont utilisés de façon courante dans l'alimentation des populations béninoises. Les résultats issus de cette recherche révèlent que les jus de boissons vendus dans les Collèges 1 et 2 de la ville d'Abomey présentent une forte charge microbienne susceptibles de provoquer en moins de 48 heures sa fermentation voire son altération. Ces jus de boisson ont également un fort pouvoir pathogène, au regard des microorganismes qui s'y retrouvent. Les principaux facteurs de contamination concernent la mauvaise qualité des intrants, les conditions malsaines de production, de conditionnement, de conservation et de vente des produits. Au vu de ces différents résultats, il est important de convier les différents acteurs de la chaine de production et de commercialisation à un aménagement approprié et à l'assainissement des sites de production et de commercialisation, ainsi qu'à un entretien régulier des matériels.

\section{References:}

1. Azonhe, T. (2009) : Analyse systémique des déterminants environnementaux de la morbidité paludique et diarrhéique chez les populations du secteur agricole dans la dépression des Tchi au sud du Bénin. Thèse de doctorat, EDP/GENV/FLASH/UAC, 238 p.

2. Baba-Moussa L., Baba-Moussa F., Bokossa I. Y., Sanni A., (2006). Etude des possibilités de contamination des aliments des rues au Bénin : cas de la ville de Cotonou, J. Rech. Sci Univ. Lomé (Togo), série A, volume 8, N², pp. 149-156. 
3. Barro, N., Cheik A. T. O., Nikiema P.A., Ouattara A. S. et Traore A. S., (2002). Evaluation de la qualité microbienne de quelques aliments de rue dans la ville de Ouagadougou au Burkina Faso. Cahiers Santé, 12 : 369-374.

4. Bryan Frank, L. ; (1994) : L'analyse des risques points critiques pour leur maîtrise, comment apprécier les risques liés à la préparation et à la conservation des aliments ? OMS - Genève, 1994.

5. Canet. C. et N'diaye C., (1996). L'alimentation de rue en Afrique. FNA/ANA, $17 \mathrm{p}$.

6. Chauliac M., Bricas N., Ategbo E., Amoussa H.W. et Zohoun I. (1998). L'alimentation hors du domicile des écoles de Cotonou (Bénin). Cahier Santé, 8 : 101-108.

7. Fayomi, B., Josse R., Adaye A., Laloe V. et Djogbe H. (1992). « Toxiinfections alimentaires en milieu rural béninois ». In Médecine d'Afrique Noire, volume 39, N5, pp.364-366.

8. Jouve, J. L., (2006). Qualité microbiologique des aliments : maitrise et critères. Polytechnica, $2^{\text {ème }}$ édition, $364 \mathrm{p}$.

9. Jouve, J. L., (1996) : Le HACCP et l'assurance de la sécurité des denrées alimentaires. In Options Qualité, N90, pp. 11-23

10. Malvy, D. et Djossou F., (2009). Les toxi-infections alimentaires collectives: aspects cliniques et épidémiologiques. Description (première partie), document d'enseignement, $13 \mathrm{p}$.

11. Monhounvedo I. (2016). Qualité sanitaire de jus de bissap (Hibiscus Sabdariffa) de fabrication locale consommé dans les établissements secondaires publics de la commune de Athiémè. Mémoire de Licence en géographie, Bénin, UAC/FLASH/DGAT, 79 p.

12. OMS (2010). Ensemble des recommandations sur la commercialisation des aliments et des boissons non alcoolisées destinés aux enfants. Rapport d'activités, Suisse, 14 p.

13. Owhe-Ureghe O. E., Ekundayo A. O., Agboniahor D. E., Oboh P. A. and Orhue P. (1993). Bacterial examination of Somme ready-to-eat foods marketed in Ekpoma, Edo state of Nigeria. Nigeria Food J., 11 : 45-52.

14. Tjoa W.S., Dupont H. L. and Sullivan P. (1977). « Location of food. Consumption and travelles diarrhea ». Am. J. Epidemiol., 106 : 61-66.

15. Umoh V. J. and Odoba M. B. (1999). Safety and quality evaluation of street foods sold in Zarian (Nigeria). Food Contr., 10 : 9-14. 